\title{
Modelling of weak quantum measurements consistent with conservation laws
}

\author{
Stanisław Sołtan ${ }^{1}$, Mateusz Frączak ${ }^{1}$, Wolfgang Belzig ${ }^{2, a}$, and Adam Bednorz ${ }^{1, b}$ \\ ${ }^{1}$ Faculty of Physics, University of Warsaw, ul. Pasteura 5, 02-093 Warsaw, Poland \\ 2 Fachbereich Physik, Universität Konstanz, 78457 Konstanz, Germany
}

Received 19 June 2020 / Accepted 5 January 2021 / Published online 1 June 2021

(C) The Author(s) 2021

\begin{abstract}
We discuss quantum mechanical detection models in the weak limit in the context of conservation laws of physical quantities. In particular, we analyze what kind of system-detector interaction can preserve the global conservation or the related symmetry, and how the final measurement on the detector affects the measured observable of the systems and its presumed conservation. It turns out that the order of noncommuting measurements results in observable differences on the level of third-order correlations functions.
\end{abstract}

\section{Introduction}

Conservation laws are important in both classical and quantum mechanics. The classical Noether theorem [1] links the conservation of certain quantities to the invariance of the dynamics of a system under specific transformations. The most prominent examples are energy conservation as a consequence of time translational invariance, conservation of momentum due to spatial translational invariance and rotational invariance implying conservation of angular momentum. In the quantum context, gauge invariance implies charge conservation and many more conservation laws are known in high-energy physics. In quantum mechanics, the observables are conserved when they commute with the Hamiltonian. Special conserved quantities called superconserved, like total charge, commute also with all observables. Hence, classical mechanics makes no difference between the conserved and superconserved case.

Conservation principles are subject to experimental verification. Neither a nonideal classical nor any quantum measurement reflects the conservation exactly. Even the smallest interaction between the system and a measuring device (detector) may cause a transfer of the conserved quantity. Coherent superposition of states with different values of a conserved quantity (e.g. energy) are allowed but not in the case of a superconserved quantity (e.g. charge), when only an incoherent mixture is allowed. Modeling of the measurement of quantities incompatible with conserved ones has been studied long ago by Wigner et al. [2-4], later discussed in the context of consistent histories [5], modular val-

\footnotetext{
a e-mail: wolfgang.belzig@uni-konstanz.de

b e-mail: adam.bednorz@fuw.edu.pl (corresponding author)
}

ues [6] and quantum clocks [7]. The quantum control of conserved quantities has become of interest recently, both experimentally and theoretically [8-10].

Objective reality means the existence of definite values of physical quantities even if they are not measured [11]. The closest approximation to not measuring at all is a weak measurement probing physical values objectively. Such a measurement is represented by weak system-detector coupling, which adds a large noise to the outcome. The noise is unavoidable if the measurement is so weak that it does not disturb the measured system but can be clearly identified and removed by a deconvolution or subtracted from correlations to get the underlying quantum statistics. This subtraction would occur also in classical noisy measurements and cannot alone dismiss the objective reality reconstructed from such measurements. However, the objectivity based on weak measurements can lead to unexpected results such as weak values [12] or the violation of the Leggett-Garg inequality $[11,13,14]$. Hence, weak quantum measurements are the closest counterpart of classical measurements so they should define objective reality and allow the verification of conservation principles.

In this paper, we define superconservation in terms of the restricted set of measurable observables that commute with a particular observable and will construct general measurements compatible with superconservation. Such measurements can change the superconserved quantity, e.g. the charge, but only in an incoherent way. However, a similar construction is in general not possible for conserved but not superconserved quantities such as energy, momentum, and angular momentum, whose conservation is apparently violated in weak measurements even if an appropriate symmetry results in a classical conservation law. The violation of conservation appears in third-order time correlations as 


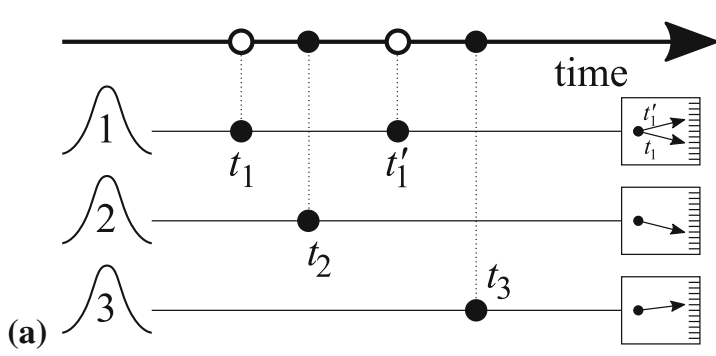

(b)

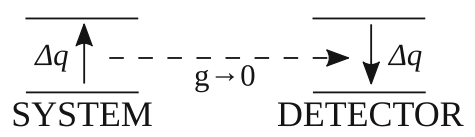

Fig. 1 a Three weak measurements. The detectors are initially independent and couple instantly to the system at $t_{1}$ (or $t_{1}^{\prime}$ ), $t_{2}$ and $t_{3}$. The conserved quantity is measured (empty circles) either at time $t_{1}<t_{2}$ or $t_{1}^{\prime}>t_{2}$. The outcomes 1 and $1^{\prime}$ inferred from the three-point correlator

we illustrate in simple model systems (Fig. 1). We also show a detection model with a conserved total Hamiltonian of system and detector, while the measured energy conservation is still violated due to the use of an autonomous quantum clock.

\section{Conservation and superconservation}

The Hermitian quantity $\hat{Q}$, defined within the system is conserved when $[\hat{H}, \hat{Q}]=0$ for the system's Hamiltonian $\hat{H}$. The $\hat{Q}$ can be superconserved if there exists a set $\mathcal{A}$ of allowed Hermitian observables such $[\hat{A}, \hat{Q}]=0$ for every $\hat{A} \in \mathcal{A}$. However, for instance, for a component of angular momentum we would have to exclude position and momentum or even the other components of angular momentum. Instead, we will distinguish quantities that are conserved but not superconserved by allowing measurement of observables not commuting with them. A typical example of a superconserved quantity is the total electric charge, where the set of observables is restricted to those that do not change the charge. This is also known as superselection rule [15]. Let us assume the decomposition of a superconserved quantity $\hat{Q}=\sum_{q} q \hat{P}_{q}$ where $\hat{P}_{q}$ are (mutually commuting) projections onto the eigenspace of the value $q$ (i.e. $\hat{Q}\left|\psi_{q}\right\rangle=q\left|\psi_{q}\right\rangle$ ). The superselection postulate says that the state of the system $\hat{\rho}$ is always an incoherent mixture $\sum_{q} \hat{P}_{q} \hat{\rho} \hat{P}_{q}$, if $\hat{Q}$ is superconserved. Then the projective measurement of $\hat{A} \in \mathcal{A}$ will not alter the $q$-eigenspace as there exists a decomposition $\hat{A}=$ $\sum_{q, a} a \hat{P}_{q a}$ with $\hat{P}_{q a}$ being the projection onto the joint eigenspace of $\hat{Q}$ and $\hat{A}$ with respective eigenvalues $q$ and $a$. If the initial state is already a $q$-eigenstate then it will remain such an eigenstate after the projection. For arbitrary measurements, positive operator-valued measures (POVM), represented by Kraus operators $\hat{K}_{c}$ (the index $c$ can represent an eigenvalue of $\hat{A}, \hat{Q}$ or both but in general it can be arbitrary) such that $\sum_{c} \hat{K}_{c}^{\dagger} \hat{K}_{c}=\hat{1}$, the state $\hat{\rho}$ will collapse to $\hat{\rho}_{c}=\hat{K}_{c} \hat{\rho} \hat{K}_{c}^{\dagger}$, normalized might differ, even for a conserved quantity in the quantum case. b Failure of conservation in the weak measurement. The transfer $\Delta q$ of the conserved quantity $q$ between the system and detector does not scale with the coupling $g$

by the probability $\operatorname{Tr} \hat{\rho}_{c}$. In principle $\hat{K}_{c}$ can act within $q$-eigenspaces, i.e. $c=q a$ and $\hat{K}_{q a}=\hat{P}_{q} \hat{K}_{a} \hat{P}_{q}$. In the most general case, the superconserving Kraus operator reads $\hat{K}_{q^{\prime} a q}=\hat{P}_{q^{\prime}} \hat{K}_{a} \hat{P}_{q}$. It means that the superconserved value can change but the system remains an incoherent mixture of $q$-eigenstates. This applies e.g. to a charge measurement in a quantum dot (which is superconserved), where the charge can leak out into an incoherent bath. The (normally) conserved quantities do not impose any additional postulates so the state can be a coherent superposition of the states of different values of energy, angular momentum, etc. A projective measurement of $\hat{A}$ which does not commute with $Q$ is enough to turn a $q$-eigenstate into a superposition. Now, if we try to postulate a POVM with superconserving Kraus operators then the actual measured average involves a linear combination of $\sum_{q q^{\prime}} \hat{P}_{q} \hat{K}_{a}^{\dagger} \hat{P}_{q^{\prime}} \hat{K}_{a} \hat{P}_{q}$ so it must commute with $\hat{Q}$ which would therefore be superconserved.

\section{Nonconservation in weak measurement}

We shall work with the established concept of quantum weak measurement [12] where

$$
\hat{K}_{a}=(2 g / \pi)^{1 / 4} \exp \left(-g(\hat{A}-a)^{2}\right)
$$

with $g \rightarrow 0$. Note that this construction is still correct in the superconserved case because $\hat{A}, \hat{K}_{a}$ and the state $\hat{\rho}$ are commuting with $\hat{Q}$ so $\hat{K}_{a}$ splits into a simple sum of $\hat{K}_{q a}$. The actual form of $\hat{K}_{a}$ can be different but the outcome is almost independent in the limit $g \rightarrow$ 0 . In the lowest order we can also neglect all $\hat{K}_{q^{\prime} a q}$. In the $g \rightarrow 0$ limit $n$-correlation of the sequence of measurements $\hat{A}, \hat{B}, \hat{C}$, reads $[16,17]$

$$
\begin{aligned}
& \langle a(t)\rangle=\langle\hat{A}(t)\rangle,\left\langle a\left(t_{1}\right) b\left(t_{2}\right)\right\rangle=\left\langle\left\{\hat{A}\left(t_{1}\right), \hat{B}\left(t_{2}\right)\right\}\right\rangle / 2 \\
& \left\langle a\left(t_{1}\right) b\left(t_{2}\right) c\left(t_{3}\right)\right\rangle=\left\langle\left\{\hat{A}\left(t_{1}\right),\left\{\hat{B}\left(t_{2}\right), \hat{C}\left(t_{3}\right)\right\}\right\}\right\rangle / 4
\end{aligned}
$$


for $t_{1}<t_{2}<t_{3}$ with the anticommutator $\{\hat{A}, \hat{B}\}=$ $\hat{A} \hat{B}+\hat{B} \hat{A}$.

The conservation in the weak measurement limit means that the measurable correlations involving the conserved quantity $q(t)$ corresponding to $\hat{Q}(t)=\hat{Q}$ will not depend on $t$. It is true at the single average, where $\langle q(t)\rangle=\langle\hat{Q}\rangle$. Interestingly, also for second order correlations the order of measurements has no influence on the result, since $\left\langle q\left(t_{1}\right) a\left(t_{2}\right)\right\rangle=\left\langle\left\{Q, A\left(t_{2}\right)\right\}\right.$ is independent of $t_{1}$. However, the situation changes for the three consecutive measurements (see Fig. 1), since in the last line of (2) the temporal order of operators matters, which has been demonstrated also experimentally [18]. Considering the difference of two measurement sequences $Q \rightarrow A \rightarrow B$ and $A \rightarrow Q \rightarrow B$, we obtain

$$
\begin{aligned}
& \left\langle\left\{\hat{Q},\left\{\hat{A}\left(t_{2}\right), \hat{B}\left(t_{3}\right)\right\}\right\}\right\rangle / 4-\left\langle\left\{\hat{A}\left(t_{2}\right),\left\{\hat{Q}, \hat{B}\left(t_{3}\right)\right\}\right\}\right\rangle / 4 \\
& \quad=\left\langle\left[\left[\hat{Q}, \hat{A}\left(t_{2}\right)\right], \hat{B}\left(t_{3}\right)\right]\right\rangle / 4 \equiv\left\langle\Delta q a\left(t_{2}\right) b\left(t_{3}\right)\right\rangle .
\end{aligned}
$$

This quantity will show up as jump at $t_{1}=t_{2}$, when measuring $\left\langle q\left(t_{1}\right) a\left(t_{2}\right) b\left(t_{3}\right)\right\rangle$. The jump will be non-zero for $Q$ not commuting with $A$ and $B$. Obviously, for superconserved quantities $Q$ (commuting with every measurable observable) the jump is absent. The violation of the conservation principle is caused by the measurement of $\hat{A}$-not commuting with $\hat{Q}$ — which allows transitions between spaces of different $q$ with the jump size $\Delta q$ not scaled by the coupling $g$, see Fig. 1 . This difference is transferred to the detector, assuming that the total quantity (of the system and detector) is conserved regardless of the system-detector interaction.

Operationally, we consider a system $S$ and a detector $D$ with the total Hamiltonian $\hat{H}+\hat{H}_{S D}$ where $\hat{H}$ acts solely within the system while $\hat{H}_{S D}$ covers all system-detector interactions, including also the detector's internal dynamics. The quantity $\hat{Q}$, defined within the system is conserved when $[\hat{H}, \hat{Q}]=0$. If additionally $\left[\hat{Q}, \hat{H}_{S D}\right]=0$ then $\hat{Q}$ is superconserved. A common superconserved example is total electric charge.

To set up $\hat{H}_{S D}$, we shall work with the established concept of quantum weak measurement [12] where the detector is coupled to the system weakly and the strong, projective measurement is applied only to the detector after decoupling. Hence, the invasiveness for the system is as small as the coupling strength. Certainly, the coupling must be nonzero, otherwise there is no measurement at all, so we need to scale the readouts with the coupling strengths. In the simplest model the detectors with the positions $x_{j}, j=1,2,3, \ldots$ are initially in Gaussian states $\left\langle x_{j} \mid \psi_{j}\right\rangle=\psi_{j}\left(x_{j}\right) \propto e^{-x_{j}^{2} / 4 \sigma}$ with the variance $\sigma$. The total system-detector Hamiltonian reads

$$
\hat{H}_{S D}=\sum_{j} g \delta\left(t-t_{j}\right) \hat{A}_{j} \hat{P}_{j}
$$

with the system's observables $\hat{A}_{j} \in\{\hat{Q}, \hat{A}, \hat{B}, \ldots\}$ and detector's momentum $\left\langle x_{j}\left|\hat{P}_{j}\right| \psi_{j}\right\rangle=-i \hbar \partial \psi_{j} / \partial x_{j}$. The initial state is a tensor product $\hat{\rho}_{S D}=\hat{\rho} \prod_{j} \hat{\rho}_{j}$ with $\hat{\rho}_{j}=\left|\psi_{j}\right\rangle\left\langle\psi_{j}\right|$. The coupling strength $g$ should be small but nonzero. After decoupling $t>t_{j}$ at the new state reads $\hat{\rho}_{S D}^{\prime}$ and one measures projectively $x_{j}$ at each detector, i.e. the probability reads $p\left(x_{1}, x_{2}, \ldots\right)=\operatorname{Tr}\left[\hat{\rho}_{S D}^{\prime} \prod_{j}\left|x_{j}\right\rangle\left\langle x_{j}\right|\right]$. From this probability distribution the observable correlations are calculated after rescaling with the coupling according to $\left\langle x_{1} x_{2} \ldots\right\rangle=\int \mathrm{d} x_{1} \mathrm{~d} x_{2} \ldots x_{1} x_{2} \ldots p\left(x_{1}, x_{2}, \ldots\right) / g_{1} g_{2} \ldots$ For the first three correlations, this procedure which yields the results (3).

\section{Total conservation}

We will now consider an extension of the above scheme, demanding the existence of a total conserved quantity. Namely, we assume there is an observable $\hat{Q}_{D}$ in the detector's space, such that $\left[\hat{Q}+\hat{Q}_{S D}, \hat{H}+\hat{H}_{S D}\right]=0$, where $\hat{H}_{S D}$ is the system-detector interaction to measure some $\hat{A}$. Suppose one measured quantity $\hat{A}$ that is noncommuting with $\hat{Q}$ (such that $[\hat{H}, \hat{Q}]=0)$ contains a term $\hat{A}_{0}=|+\rangle\langle-|+|-\rangle\langle+|$ where $| \pm\rangle$ are eigenstates of $\hat{Q}$ with the eigenvalues $\tilde{q}_{ \pm}$such that $\tilde{q}_{+}-\tilde{q}_{-}=q>0$. We introduce detector states that are eigenstates of $\hat{Q}_{D}$ enumerated by $\left|q_{i}\right\rangle$ with eigenvalues $q_{i}$. The interaction keeping $\hat{Q}+\hat{Q}_{D}$ conserved must have the form

$$
\hat{H}_{S D}=\alpha\left(\left|+q_{0}\right\rangle\left\langle-q_{+}|+|-q_{0}\right\rangle\left\langle+q_{-}\right|-\text {h.c. }\right),
$$

where $\left| \pm q_{i}\right\rangle$ denotes a product state of the system's states $| \pm\rangle$ and the detector's states $\left|q_{+}\right\rangle,\left|q_{0}\right\rangle,\left|q_{-}\right\rangle$with $q_{+}=q, q_{0}=0, q_{-}=-q$. Initially the detector is in the state $\left|q_{0}\right\rangle$, while $\alpha_{0+}=\alpha_{-0}=g \hbar \delta\left(t-t_{0}\right) / \sqrt{2}$ for the measurement at $t=t_{0}$. Afterwards, we project the detector on the orthonormal basis states $|0\rangle=$ $\left(\left|q_{+}\right\rangle-\left|q_{-}\right\rangle\right) / \sqrt{2}$ and $| \pm 1\rangle=\left(\left|q_{+}\right\rangle+\left|q_{-}\right\rangle\right) / 2 \mp i\left|q_{0}\right\rangle / \sqrt{2}$. Note that these states, $|0, \pm 1\rangle$, have two important properties, $\left\langle\hat{Q}_{D}\right\rangle=0$ while $\left\langle\hat{Q}_{D}^{2}\right\rangle>0$ for each of them, which means they are not biased by $Q_{D}$ but still they are not eigenstates of $\hat{Q}_{D}$. We could use other orthonormal states of the same properties. Projecting onto eigenstates of $\hat{Q}_{D}$ would never give the outcome related to $\hat{A}$ as follows from the Wigner-Araki-Yanase theorem [2-4]. Assigning values to the outcome states $x=0, \pm 1$, the average $x$ and the new system's state read

$$
\begin{aligned}
\langle x\rangle= & \operatorname{Tr} \sum_{x} x\left\langle x\left|\hat{\rho}_{S D}^{\prime}\right| x\right\rangle=g \operatorname{Tr} \hat{A}_{0} \hat{\rho}+O\left(g^{3}\right), \\
\hat{\rho}_{S}^{\prime \prime}= & \sum_{x}\left\langle x\left|\hat{\rho}_{S D}^{\prime}\right| x\right\rangle=\hat{\rho} \\
& -g^{2}\left[\hat{A}_{0},\left[\hat{A}_{0}, \hat{\rho}\right]\right] / 2+O\left(g^{4}\right) .
\end{aligned}
$$


Here, $\hat{\rho}_{S D}^{\prime}$ is the system-detector state after the interaction (in $S D$-space) and $\hat{\rho}_{S}^{\prime \prime}$ is the system's reduced density matrix after tracing out the detector. The Tr is taken only in the system space. To derive relations like (2) for correlations of $x$ with the outcome $b$ of a subsequent measurement of $\hat{B}$ we obtain

$$
\langle b x\rangle=g \operatorname{Tr} \hat{B}\left\{\hat{A}_{0}, \hat{\rho}\right\} / 2+O\left(g^{3}\right) .
$$

That we used discrete weak measurement is not essential because in the limit of weak coupling the discreteness is irrelevant.

The model discussed above can be replaced by a Gaussian detector consisting of a pair of oscillators with $\left[\hat{a}_{i}, \hat{a}_{j}^{\dagger}\right]=\delta_{i j}$ originally in the ground state $|\Omega\rangle$ $\left(\hat{a}_{i}|\Omega\rangle=0\right)$ and interaction

$$
\begin{aligned}
\hat{H}_{S D}= & q\left(\hat{a}_{1}^{\dagger} \hat{a}_{1}+\hat{a}_{2}^{\dagger} \hat{a}_{2}\right)+i g|+\rangle \\
& \times\langle-|\left(\hat{a}_{1}-\hat{a}_{2}^{\dagger}\right) \hbar \delta\left(t-t_{0}\right) / \sqrt{2}+\text { h.c. }
\end{aligned}
$$

while measuring the combined position $\hat{X}=\hat{X}_{1}+\hat{X}_{2}$ immediately after $t_{0}$ (otherwise $\hat{X}_{1,2}$ will oscillate) with $\sqrt{2} \hat{X}_{i}=\hat{a}_{i}+\hat{a}_{i}^{\dagger}$ at the detectors' part. By mapping on the eigenstates of the conserved quantity $\hat{Q}_{D}$, viz., $\left|q_{0}\right\rangle \rightarrow|\Omega\rangle,\left|q_{+}\right\rangle \rightarrow-i \hat{a}_{1}^{\dagger}|\Omega\rangle$ and $\left|q_{-}\right\rangle \rightarrow-i \hat{a}_{2}^{\dagger}|\Omega\rangle$, we obtain the same result (6) replacing $x$ by eigenstates of $\hat{X}$ because

$$
\langle x\rangle=\operatorname{Tr} \hat{X} \hat{\rho}^{\prime},\langle b x\rangle=\operatorname{Tr} \hat{B} \hat{X} \hat{\rho}^{\prime}
$$

with

$$
\begin{aligned}
\hat{\rho}^{\prime}= & \hat{\rho}|0\rangle\langle 0|-i g|+\rangle\left\langle-|\hat{\rho}| q_{-}\right\rangle \\
& \times\left\langle q_{0}|/ \sqrt{2}-i g|-\right\rangle\left\langle+|\hat{\rho}| q_{+}\right\rangle\left\langle q_{0}\right| / \sqrt{2}+\text { h.c. }
\end{aligned}
$$

in the lowest order of $g$. One could take even a single oscillator

$$
\hat{H}_{S D}=q \hat{a}^{\dagger} \hat{a}+i g|+\rangle\langle-| \hat{a} \hbar \delta\left(t-t_{0}\right) / \sqrt{2}+\text { h.c. }
$$

but then the initial detector's state must be squeezed, with $\left\langle\hat{X}^{2}\right\rangle=\sigma \ll g$ (i.e. it is not a ground state) and $\langle\{\hat{X}, \hat{P}\}\rangle=0$ for $\sqrt{2} \hat{P}=i\left(\hat{a}-\hat{a}^{\dagger}\right)$. Then the we get approximately (2) with the result corrected by $\sim \sigma$ and invasiveness $\hat{\rho}^{\prime \prime}-\hat{\rho} \sim g^{2} / \sigma$. For sequential measurements one needs to couple and decouple each detector in a sequence. Although our model respects the conservation of the total quantity, the energy eigenstates of the detector and the system get entangled during the interaction. Now, the projection inside the detector on the non-eigenstates of $\hat{Q}_{S D}$ affects the system due to the entanglement with the detector even when they are already decoupled.

When $\hat{H}=\hat{Q}$ the above model, involving instant interaction, is in conflict with time-translation symmetry. We shall deal with this problem in the next section.

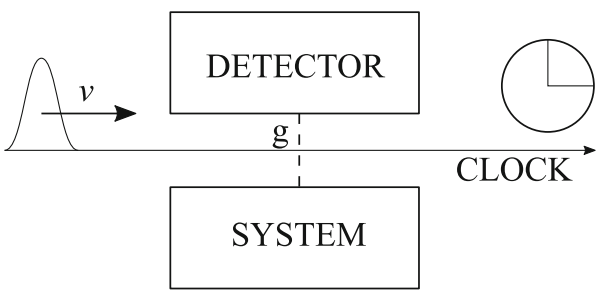

Fig. 2 Detection model based on a clock. The clock is a localized particle traveling with a constant speed $v$. The interaction between the detector and system takes place only when the clock is passing the interaction point

\section{Time-translational symmetry}

Performing a series of measurements already breaks time-translational symmetry and the total energy is not necessarily conserved. However, one can keep the time symmetry by replacing the detector-system interaction by a clock-based detection scheme [7], effectively moving the breaking of symmetry from time to space axis, see Fig. 2. The total Hamiltonian reads

$$
\hat{H}+\hat{H}_{x}+\hat{H}_{z}+\hat{H}_{I}
$$

where $\hat{H}$ is the system's part, $\hat{H}_{x}$ - the detector's part, $\hat{H}_{z}$ - the clock's part, and finally $\hat{H}_{I}$ is the interaction between the clock, the system and detector. Each part is time-independent so the time translation symmetry is preserved. Both, the detector and the clock can be represented by single real variables, $x$ and $z$. Now, to measure the system's $\hat{A}$ at time $t_{1}$ we set $\hat{H}_{x}=0$ and

$$
\hat{H}_{z}=v \hat{p}_{z}, \hat{H}_{I}=g \hat{A} \delta(\hat{z}) \hat{p}_{x}
$$

where $\hat{p}_{x, z}$ are conjugate (momenta), i.e. $\hat{p}_{x}=-i \hbar \partial / \partial x$ and $g \rightarrow 0$ is a weak coupling constant. The initial state $($ at $t=0)$ reads $\hat{\rho} \hat{\rho}_{x} \hat{\rho}_{z}$, where both $\hat{\rho}_{x, z}=\left|\psi_{x, z}\right\rangle\left\langle\psi_{x, z}\right|$ are taken as Gaussian states

$$
\begin{aligned}
& \psi_{z}(z)=(2 \pi \sigma)^{-1 / 4} \exp \left(-\left(z+v t_{1}\right)^{2} / 4 \sigma\right), \\
& \psi_{x}(x)=(\pi / 2)^{-1 / 4} \exp \left(-x^{2}\right),
\end{aligned}
$$

respectively. For small $g$ and $\sigma$ the interaction effectively occurs at time $t=t_{1}$ and, in the end (after the clock decouples the system and the detector again) to lowest order we find

$$
\langle x\rangle \simeq g\langle\hat{A}\rangle=g\left\langle a\left(t_{1}\right)\right\rangle .
$$

The clock, detector, and the system are formally constantly coupled but we have to set the initialization of the detector before $t_{1}$ and readout after $t_{1}$ although the precise times are irrelevant. For sequential measurements one simply adds more independent detectors and clocks, obtaining in the lowest order of $g$

$$
\left\langle x_{A} x_{B}\right\rangle \simeq g^{2}\left\langle a\left(t_{1}\right) b\left(t_{2}\right)\right\rangle,
$$




$$
\begin{aligned}
& \left\langle x_{A} x_{B} x_{C}\right\rangle \simeq g^{3}\left\langle a\left(t_{1}\right) b\left(t_{2}\right) c\left(t_{3}\right)\right\rangle, \\
& \left\langle x_{A} x_{B} x_{C} x_{D}\right\rangle \simeq g^{4}\left\langle a\left(t_{1}\right) b\left(t_{2}\right) c\left(t_{3}\right) d\left(t_{4}\right)\right\rangle,
\end{aligned}
$$

with the right hand sides given by the quantum expressions (2). It follows that if the energy of the system is observed ( $\hat{H}$ is the first observable) then in the limit of weak measurement it commutes with any single measurement-including the measurement of the energy in the clock $\left(\hat{H}_{z}\right.$ is the second observable). However it stops commuting when more measurements are involved.

As is suggested below, the clock could actually be realized as an additional degree of freedom of the measured system. Furthermore, as $\hat{H}_{x}=0$, effectively there is no leakage of energy to the detector. Thus, as suggested in [7], it may be some transfer of the energy to the clock. This point qualifies for further investigation.

Although the above detection model is based on timeinvariant dynamics, the initial state of the clock spoils the symmetry. The time-invariant state would require a constant flow of particles, see [19] for detailed construction. However, such a constant interaction between the detector (clock) and the system leads to a backaction and makes the measurement invasive e.g. by heating or cooling. In that case, to keep the system in a stationary state, it would need an additional coupling to a heat bath.

\section{Examples}

The examples below illustrate the fundamental finding of our article. If one tries to verify the conservation of energy or other conserved quantity while measuring other observable not commuting with the Hamiltonian, it is possible to find a violation of the conservation. It constitutes a pure quantum effect since it vanishes at high temperature, where the classically expected conservation holds.

Examples 6.1 and 6.2 are for the energy nonconservation. One could object that performing a series of measurements already breaks time-translational symmetry and therefore energy is not conserved. We will illustrate in the following that this is not the case by considering other conserved observable - angular momentum in a rotationally invariant system-in Sect. 6.3. This one also provides a proposition of experimental test.

\subsection{Two-level atom}

As an example we can take the basic two-level system $(| \pm\rangle$ basis) with the Hamiltonian $\hat{H}=\hat{Q}=\hbar \omega|+\rangle\langle+|$ and $\hat{A}=\hat{B}=\hat{X}=|+\rangle\langle-|+|-\rangle\langle+|$. Then, with $\omega>0$ the ground state is $|-\rangle$ and the third order correlation $\left\langle h\left(t_{1}\right) x\left(t_{2}\right) x\left(t_{3}\right)\right\rangle$ at zero temperature for $t_{3}>t_{1,2}$ reads $\hbar \omega\left(1-\theta\left(t_{2}-t_{1}\right)\right) \cos \left(\omega\left(t_{2}-t_{3}\right)\right) / 2$. The jump is $\langle\Delta h x(0) x(\tau)\rangle=\hbar \omega \cos (\omega \tau) / 2$. The result can be gen-

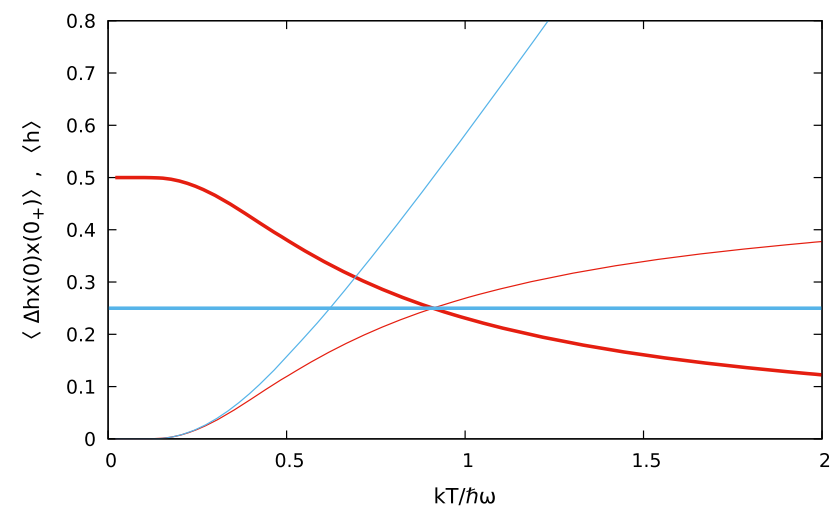

Fig. 3 The non-conserving jump for $\tau=0^{+}$(thick lines) compared to the average energy (thin lines) for the twolevel system (red) and the harmonic oscillator (blue). At high temperatures the jump becomes unobservable and the classical conservation is restored. All quantities are normalized to $\hbar \omega$

eralized to finite temperature

$$
\langle\Delta h x(0) x(\tau)\rangle=\hbar \omega \cos (\omega \tau) \tanh (\hbar \omega / 2 k T) / 2 .
$$

For increasing temperature the jump diminishes as illustrated in Fig. 3.

\subsection{Harmonic oscillator}

Another basic example is the harmonic oscillator with $\hat{H}=\hat{Q}=\hbar \omega \hat{a}^{\dagger} \hat{a}$ with $\left[\hat{a}, \hat{a}^{\dagger}\right]=1$. Taking the dimensionless position $\sqrt{2} \hat{X}=\hat{a}^{\dagger}+\hat{a}=\hat{A}=\hat{B}$, we find for the jump $\langle\Delta h x(0) x(t)\rangle=-\hbar \omega \cos (\omega t) / 4$ independent of the state of the system. As illustrated in Fig. 3, the jump becomes unobservable at high temperatures since the average energy $\langle h\rangle=\hbar \omega /[\exp (\hbar \omega / k T)-1]$ increases with temperature.

\subsection{Angular momentum conservation}

We propose an experiment to demonstrate the failure of a conservation principle for angular momentum in third order correlations in weak measurements. Instead of energy we consider one component of angular momentum, say $\hat{L}_{z}=\hat{X} \hat{P}_{y}-\hat{Y} \hat{P}_{x}$, which gives rise to a magnetic moment $\sim \mu_{B} L_{z}$ of the order a Bohr magneton $\mu_{B}$. The magnetic field generated by this moment can in principle be detected by a sensitive magnetometer (e.g. a superconducting quantum interferometer device, SQUID). The other two observables will be the particle's positions $\hat{X}$ and $\hat{Y}$ which can be measured e.g. by the voltage of a capacitor depending linearly on $x$ and $y$ for small changes in position, see the setup sketch in Fig. 4. The two positions $x$ and $z$ will be measured at times $t_{2}$ and $t_{3}$, respectively.

The quantity of matter is $\left\langle l_{z}\left(t_{1}\right) x\left(t_{2}\right) y\left(t_{3}\right)\right\rangle$. Suppose the particle is in a harmonic trap rotationally invariant about $z$ axis. The $x y$ part of the trap Hamiltonian reads 


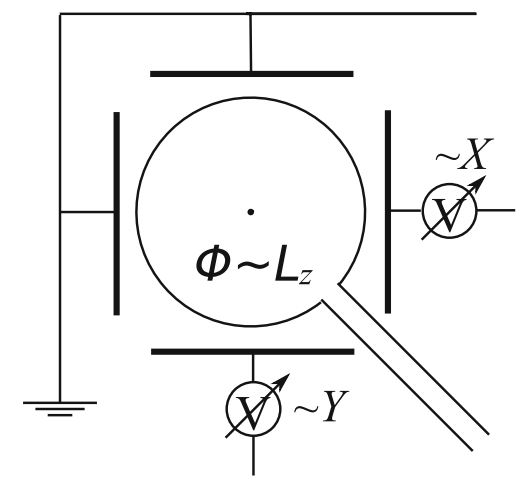

Fig. 4 A trap for a charged particle that is invariant under rotation about $z$ axis. The angular momentum in $z$ direction corresponding the magnetic field generated by the particle is measured by SQUID. Orthogonal capacitors measure the position in the $x y$-plane

$\hat{H}_{\perp}=\hbar \omega\left(\hat{a}_{x}^{\dagger} \hat{a}_{x}+\hat{a}_{y}^{\dagger} \hat{a}_{y}\right)$, with $\left[\hat{a}_{x, y}, \hat{a}_{x, y}^{\dagger}\right]=1,\left[\hat{a}_{x}, \hat{a}_{y}\right]=$ $\left[\hat{a}_{x}^{\dagger}, \hat{a}_{y}\right]=0$. Then $\sqrt{2} \hat{X}=\hat{a}_{x}^{\dagger}+\hat{a}_{x}$ and $\sqrt{2} \hat{P}_{x} / i \hbar=\hat{a}_{x}^{\dagger}-$ $\hat{a}_{x}$ (rescaled by a length unit), similarly for $y$, and $\hat{L}_{z}=$ $i \hbar\left(\hat{a}_{x} \hat{a}_{y}^{\dagger}-\hat{a}_{y} \hat{a}_{x}^{\dagger}\right)$. In the ground state $|0\rangle$ we have $\hat{L}_{z}|0\rangle=$ 0 so only $\left\langle\hat{Y}\left(t_{3}\right) \hat{L}_{z}\left(t_{1}\right) \hat{X}\left(t_{2}\right)\right\rangle$ and $\left\langle\hat{X}\left(t_{2}\right) \hat{L}_{z}\left(t_{1}\right) \hat{Y}\left(t_{3}\right)\right\rangle$ contribute in (2). These terms can appear only when $t_{2}<t_{1}$ or $t_{3}<t_{1}$. We find

$$
\begin{aligned}
\left\langle l_{z}(\right. & \left.\left.t_{1}\right) x\left(t_{2}\right) y\left(t_{3}\right)\right\rangle \\
= & \left(1-\theta\left(t_{2}-t_{1}\right) \theta\left(t_{3}-t_{1}\right)\right) \\
& \times\left\langle\hat{X}\left(t_{2}\right) \hat{L}_{z}\left(t_{1}\right) \hat{Y}\left(t_{3}\right)+\hat{Y}\left(t_{3}\right) \hat{L}_{z}\left(t_{1}\right) \hat{X}\left(t_{2}\right)\right\rangle / 4 \\
= & {\left[1-\theta\left(t_{2}-t_{1}\right) \theta\left(t_{3}-t_{1}\right)\right] \hbar \sin \left[\omega\left(t_{2}-t_{3}\right)\right] / 4 }
\end{aligned}
$$

The jump is therefore given by

$$
\left\langle\Delta l_{z} x\left(t_{2}\right) y\left(t_{3}\right)\right\rangle_{0}=\hbar \sin \left[\omega\left(t_{2}-t_{3}\right)\right] / 4
$$

and is again state-independent. Hence, the finite jump illustrates that the angular momentum conservation is apparently violated by this experiment. At finite temperature $T$ for $t_{1}<t_{2,3}$, the correlator $\left\langle l_{z<x} x\left(t_{2}\right) y\left(t_{3}\right)\right\rangle=$ $\hbar \sin \left[\omega\left(t_{2}-t_{3}\right)\right] / 4 \sinh ^{2}\left(\hbar \omega / 2 k_{B} T\right)$ increases with temperature and makes the (temperature-independent) jump unobservable.

Since in this setup the detectors are coupled permanently, a frequency-domain measurement might be more appropriate. In frequency domain the observables are $A(\alpha)=\int \mathrm{d} t e^{i \omega t} A(t)$. Taking all our previous arguments to frequency domain, the conservation of a quantity $\hat{Q}(\alpha)$ means that correlators vanish for $\alpha \neq 0$. Interestingly, transforming to frequency domain we find at zero temperature and for $\gamma, \alpha, \beta \neq 0$ that

$$
\left\langle l_{z}(\gamma) x(\alpha) y(\beta)\right\rangle=\frac{i \pi \hbar \omega(\alpha-\beta) \delta(\gamma+\alpha+\beta)}{2\left(\alpha^{2}-\omega^{2}\right)\left(\beta^{2}-\omega^{2}\right)}
$$

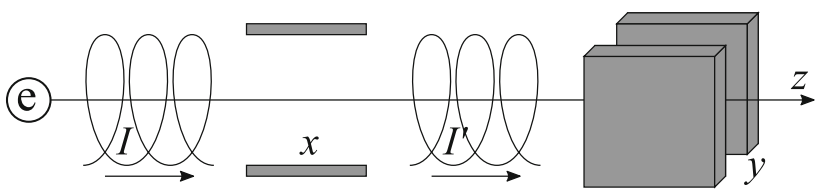

Fig. 5 A charged particle (e.g. electron) goes along the tube with angular momentum measured by the currents in either of two coils, $I$ or $I^{\prime}$. Meanwhile, the position in $x$ and $y$ direction is measured by two perpendicular capacitors

Clearly, the conservation principle for angular momentum is violated by (20). Hence, either by time- or frequency-resolved measurements one should see experimentally the nonconservation of angular momentum.

To realize a time-resolved measurement, we suggest to test the angular momentum conservation with a charge moving inside a round tube along $z$ direction, similar to the recent test of the order of measurements [18]. In the simplest model take $\hat{H}=\hat{H}_{z}+\hat{H}_{\perp}$ and we keep the same harmonic potential in the $x y$ plane as above and add some $\hat{H}_{z}=v \hat{p}_{z}$ with velocity $v$ (like the clock form the previous section). Preparing a wavepacket as a product of the ground state of $\hat{H}_{\perp}$ and $\psi(z)$ of sufficiently short width, we can measure essentially the same quantity (18) by putting a sequence of weak detectors along the tube, see Fig. 5. The angular momentum can be measured by the current signature in the coil, like in the recent experiment [8]. We simplify the coil-electron beam interaction to $\lambda(z) \hat{I} \hat{L}_{z}$ where $\lambda$ only non-zero inside the coil and $\hat{I}$ is the current operator in the coil. Similarly, the measurement of $x$ and $y$ can be modeled by local capacitive couplings. In this way, the measurement times are translated into position according to $t=z / v$, just like in the time-invariant energy detection in the previous section. The jump (19) can then be detected by placing the coil at two different positions, see Fig. (5).

As regards the rotational invariance of the system, detection of $X$ and $Y$ can be performed by the detectorsystem coupling

$$
\hat{H}_{I}=g \delta(\hat{z})\left(\hat{X} \hat{p}_{x D}+\hat{Y} \hat{p}_{y D}\right)
$$

with the initial state $\hat{\rho} \hat{\rho}_{x, y}^{D}$ and the detector's state $\hat{\rho}_{x, y}^{D}=|\psi\rangle\langle\psi|$,

$$
\psi\left(x_{D}, y_{D}\right)=(\pi / 2)^{-1 / 2} \exp \left(-\left(x_{D}^{2}+y_{D}^{2}\right)\right)
$$

Then both the interaction and the initial state of the system and detector are rotationally invariant so the total angular momentum is conserved. Only the readout, either $x$ or $y$ of the detector, prefers one direction, i.e.

$$
\left\langle x_{D}\right\rangle \simeq g\langle x\rangle
$$

with straightforward generalization to sequential measurements like (16). 


\section{Conclusions}

We have shown that conservation laws in quantum mechanics need to be considered with care since their experimental verification might depend on the measurement context even in the limit of weak measurements. We can distinguish superconserved observables, which will be conserved whatever measurement will be performed, which as consequence are subject to superselection rules - excluding superpositions of eigenstates with different eigenvalues. In contrast, usual conserved quantities like energy or angular momentum might be found to be non-conserved if measured in the context of other non-commuting variables. We have defined an operational criterion to detect the non-conservation by third-order correlation functions. The non-conservation can also be formulated as Leggett-Garg-type test showing the connection to the absence of macrorealism in quantum mechanics. In future, it might be interesting on one hand to study more realistic scenarios for quantum measurements taking into account decoherence or more general detectors [20].

Acknowledgements We thank for financial support by the DFG through SFB 767 (WB) and discussions with N. Gisin and E. Karimi. S.S. and M.F. made calculation for the examples, W.B. and A.B. wrote the general scheme and discussion.

Funding Information Open Access funding enabled and organized by Projekt DEAL.

Open Access This article is licensed under a Creative Commons Attribution 4.0 International License, which permits use, sharing, adaptation, distribution and reproduction in any medium or format, as long as you give appropriate credit to the original author(s) and the source, provide a link to the Creative Commons licence, and indicate if changes were made. The images or other third party material in this article are included in the article's Creative Commons licence, unless indicated otherwise in a credit line to the material. If material is not included in the article's Creative Commons licence and your intended use is not permitted by statutory regulation or exceeds the permitted use, you will need to obtain permission directly from the copyright holder. To view a copy of this licence, visit http://creativecomm ons.org/licenses/by/4.0/.

\section{References}

1. E. Noether, Invariante Variationsprobleme, Nachr. d. König. Gesellsch. D. Wiss. zu Göttingen, Math. Phys. Klasse. 235 (1918)

2. E.P. Wigner, Die messung quantenmechanischer operatoren. Z. Phys. 131, 101 (1952)

3. H. Araki, M.M. Yanase, Measurement of quantum mechanical operators. Phys. Rev. 120, 622 (1960)

4. M.M. Yanase, Optimal measuring apparatus. Phys. Rev. 123, $666(1961)$
5. J.B. Hartle, R. Laflamme, D. Marolf, Conservation laws in the quantum mechanics of closed systems. Phys. Rev. D 51, 7007 (1995)

6. Y. Aharonov, S. Popescu, D. Rohrlich, On conservation laws in quantum mechanics. Proc. Nat. Acad. Sci. 118, e1921529118 (2021)

7. N. Gisin, E.Z. Cruzeiro, Quantum measurements, energy conservation and quantum clocks. Ann. Phys. 530, 1700388 (2018)

8. H. Larocque, F. Bouchard, V. Grillo, A. Sit, S. Frabboni, R.E. Dunin-Borkowski, M.J. Padgett, R.W. Boyd, E. Karimi, Nondestructive measurement of orbital angular momentum for an electron beam. Phys. Rev. Lett. 117, 154801 (2016)

9. S.M. Lloyd, M. Babiker, G. Thirunavukkarasu, J. Yuan, Electron vortices: beams with orbital angular momentum. Rev. Mod. Phys. 89, 035004 (2017)

10. K.Y. Bliokh et al., Theory and applications of freeelectron vortex states. Phys. Rep. 690, 1 (2017)

11. A.J. Leggett, A. Garg, Quantum mechanics versus macroscopic realism: is the flux there when nobody looks? Phys. Rev. Lett. 54, 857 (1985)

12. Y. Aharonov, D.Z. Albert, L. Vaidman, How the result of a measurement of a component of the spin of a spin$1 / 2$ particle can turn out to be 100 . Phys. Rev. Lett. 60, 1351 (1988)

13. C. Emary, N. Lambert, F. Nori, Leggett-Garg inequalities. Rep. Prog. Phys. 77, 016001 (2014)

14. A. Palacios-Laloy, F. Mallet, F. Nguyen, P. Bertet, D. Vion, D. Esteve, A.N. Korotkov, Experimental violation of a Bell's inequality in time with weak measurement. Nat. Phys. 6, 442 (2010)

15. S.D. Bartlett, T. Rudolph, R.W. Spekkens, Reference frames, superselection rules, and quantum information. Rev. Mod. Phys. 79, 555 (2007)

16. A. Bednorz, W. Belzig, Quasiprobabilistic interpretation of weak measurements in mesoscopic junctions. Phys. Rev. Lett. 105, 106803 (2010)

17. L. Plimak, S. Stenholm, Causal signal transmission by quantum fields. V: Generalised Keldysh rotations and electromagnetic response of the Dirac sea. Ann. Phys. (N.Y.) 327, 2691 (2012)

18. D. Curic, M.C. Richardson, G.S. Thekkadath, J. Florez, L. Giner, J.S. Lundeen, Experimental investigation of measurement-induced disturbance and time symmetry in quantum physics. Phys. Rev. A 97, 042128 (2018)

19. A. Bednorz, W. Belzig, Models of mesoscopic timeresolved current detection Phys. Rev. B 81, 125112 (2010)

20. J. Bülte, A. Bednorz, C. Bruder, W. Belzig, Noninvasive quantum measurement of arbitrary operator order by engineered non-Markovian detectors. Phys. Rev. Lett. 120, 140407 (2018) 